\title{
Final implementation, commissioning, and performance of embedded collimator beam position monitors in the Large Hadron Collider
}

\author{
Gianluca Valentino, ${ }^{1,2, *}$ Guillaume Baud, ${ }^{1}$ Roderik Bruce, ${ }^{1}$ Marek Gasior, ${ }^{1}$ Alessio Mereghetti, ${ }^{1}$ \\ Daniele Mirarchi, ${ }^{1}$ Jakub Olexa, ${ }^{1}$ Stefano Redaelli, ${ }^{1}$ Belen Salvachua, ${ }^{1}$ \\ Alessandra Valloni, ${ }^{1}$ and Jorg Wenninger ${ }^{1}$ \\ ${ }^{1}$ CERN, CH-1211 Geneva 23, Switzerland \\ ${ }^{2}$ University of Malta, Msida MSD2080, Malta \\ (Received 21 April 2017; published 10 August 2017)
}

\begin{abstract}
During Long Shutdown 1, 18 Large Hadron Collider (LHC) collimators were replaced with a new design, in which beam position monitor (BPM) pick-up buttons are embedded in the collimator jaws. The BPMs provide a direct measurement of the beam orbit at the collimators, and therefore can be used to align the collimators more quickly than using the standard technique which relies on feedback from beam losses. Online orbit measurements also allow for reducing operational margins in the collimation hierarchy placed specifically to cater for unknown orbit drifts, therefore decreasing the $\beta^{*}$ and increasing the luminosity reach of the LHC. In this paper, the results from the commissioning of the embedded BPMs in the LHC are presented. The data acquisition and control software architectures are reviewed. A comparison with the standard alignment technique is provided, together with a fill-to-fill analysis of the measured orbit in different machine modes, which will also be used to determine suitable beam interlocks for a tighter collimation hierarchy.
\end{abstract}

DOI: 10.1103/PhysRevAccelBeams.20.081002

\section{INTRODUCTION}

The Large Hadron Collider (LHC) [1] at CERN in Geneva, Switzerland is at the particle accelerator technology frontier, with a stored beam energy higher than any previous collider, having achieved around $270 \mathrm{MJ}$ so far out of a design value of 362 MJ. A solid, reliable and complex collimation system [2] is installed in the LHC to protect the machine against unavoidable beam losses by cleaning high-energy halo particles before they can heat the superconducting magnets and quench them. In addition, collimators protect the aperture from single-turn beam losses, which may occur if the beams are miskicked during injection or dump.

Collimation is required at all phases of the LHC machine cycle, from beam injection to the energy ramp to the current $6.5 \mathrm{TeV}$ (7 TeV nominal energy), at flat top, while reducing the beam size in the experimental regions (squeeze), when bringing the beams into collisions (adjust), and during the ensuing physics (stable beams). In order to maintain optimum cleaning performance at all times, the two jaws of each collimator are placed parallel to and equidistant from the beam at the desired number of beam $\sigma$ units.

*gianluca.valentino@cern.ch

Published by the American Physical Society under the terms of the Creative Commons Attribution 4.0 International license. Further distribution of this work must maintain attribution to the author(s) and the published article's title, journal citation, and DOI.
The collimators are positioned to form a multistage hierarchy, with the primary collimators (TCP) closest to the beam, followed by the secondary collimators (TCSG) and absorbers (TCLA). Tertiary collimators (TCT) are installed to protect the experimental regions. Most of the 108 LHC collimators are installed in Insertion Region (IR) 3 and IR7 to clean particles with large off-momentum and betatron offsets respectively.

Beam-based collimator alignment [3] is used to determine the beam center and beam size at each collimator, from which the operational settings can be calculated. An established technique for aligning a collimator involves moving each jaw in steps of 5-20 $\mu \mathrm{m}$ towards the beam until a spike is observed in the beam loss signal of a Beam Loss Monitor (BLM) ionization chamber [4] positioned directly downstream of the collimator. Although efforts were made to optimize this procedure as much as possible in terms of time required and automation [5-7], the time required to align collimators remains a limitation to the operational efficiency of the LHC, in particular the yearly recommissioning after long stops and the configuration changes around experiments, which affect the orbit at the tertiary collimators. A direct measurement of the beam orbit at the collimator via an embedded beam position monitor (BPM) would allow for faster alignment, as well as to be able to respond more quickly to machine configuration changes in the experimental insertion regions (IRs), for example of the crossing angle or $\beta^{*}$ (the $\beta$-function at the collision points).

The presence of in-jaw BPMs allows for a safer alignment procedure as the collimator would no longer need to 
touch the beam, thus eliminating the risk of jaw damage. The method is noninvasive, and therefore can be done with beam intensities higher than those for which the beam can be approached by the jaw in the standard BLM-based alignment $\left(3 \times 10^{11} \mathrm{p}\right.$ at $\left.7 \mathrm{TeV}\right)$. Alignment of all collimators in parallel is fully possible as the beam is not touched and therefore there are no crosstalk effects due to beam losses. Thanks to the continuous reading, it is also possible to keep the jaws aligned during the dynamic phases of the LHC machine cycle. A further motivation for having embedded collimator BPMs is to reduce the orbit margin in the cleaning hierarchy. As the orbit at the collimators will be more precisely known, the present margins used for the $\beta^{*}$-reach calculation [8,9] could potentially be reduced if the beam orbit is very stable, providing more room to squeeze the $\beta^{*}$ and increase the luminosity. The BPMs could also be used to better protect the TCTs and triplet magnets thanks to a beam interlock on the measurement that would dump the beams if the orbit at the TCT exceeded a safe threshold.

Several beam studies were successfully performed in the Super Proton Synchrotron (SPS) to determine the feasibility of the design $[10,11]$ as well as to test a successive approximation alignment algorithm and related control software $[12,13]$. This paved the way for the replacement in the LHC of 16 TCTs in the experimental IRs and the 2 TCSGs in IR6 in Long Shutdown 1 (LS1) in 2013-2015 with new collimators with embedded BPM pick-ups [14]. The new collimators with pick-ups are referred to as TCTP and TCSP respectively. The pick-ups are installed on the upstream and downstream ends of the copper-based tapered region of each jaw [15], as shown in Fig. 1. They are retracted by $8.5 \mathrm{~mm}$ and $8.6 \mathrm{~mm}$ from the active surfaces of the TCSP and TCTP, respectively, to protect them from possible beam impacts.

In this paper, the results from beam commissioning and deployment in standard LHC operation in 2015 and 2016 of the embedded BPM collimators are reported. First, an

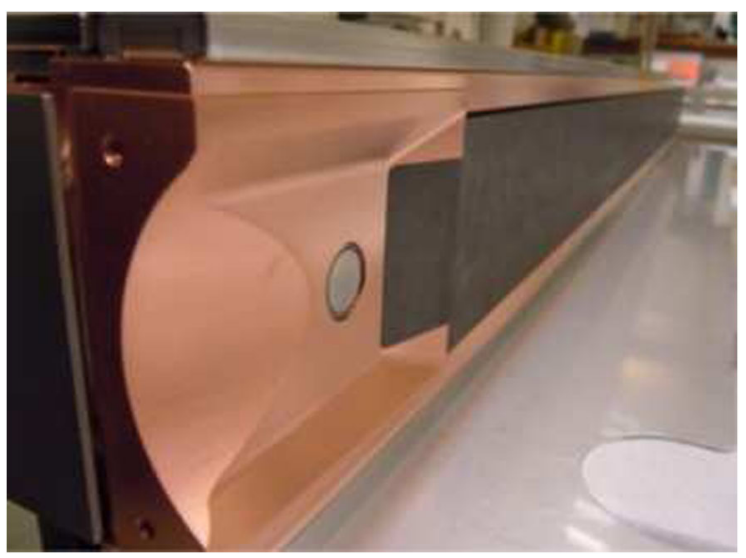

FIG. 1. View of one end of a TCSP collimator jaw showing the circular BPM pick-up button embedded in the taper. overview of the data acquisition and control system is provided, followed by a presentation of characterization measurements of the embedded BPMs for determination of calibration and nonlinearity corrections. Results from BPM-based alignment and comparison to the established BLM-based technique are presented. This is followed by operational performance results and their implications, namely the fill-to-fill performance of the BPMs over the 2016 LHC proton run, the exploitation of a direct measurement of the beam orbit at the collimators to produce more accurate collimator settings for the dynamic parts of the LHC machine cycle, and the determination of suitable interlock thresholds to reduce the existing collimation hierarchy margins and therefore push the performance of the LHC even further.

\section{DATA ACQUISITION AND CONTROL SYSTEM ARCHITECTURE}

\section{A. Data acquisition system}

The BPM data are acquired from the button pick-ups via the Diode ORbit and OScillation (DOROS) system [16], which was developed following tests in the lab and the SPS $[17,18]$. DOROS converts the short BPM electrode pulses into slowly varying signals using compensated diode detectors, whose output signals are then precisely processed and acquired using 24-bit analog-to-digital converters. This allows for a sub-micrometer orbit resolution to be achieved by performing measurements over thousands of machine revolutions. Each front-end process signals from one BPM electrode pair. An automatic gain control mechanism is implemented in a FPGA to ensure that the signals remain within a fixed linear range independently of the BPM aperture, which varies for different machine configurations for the case of the collimator BPMs, or the beam intensity in the LHC.

\section{B. Control system architecture}

The data are sent from the DOROS front-ends in the form of User Datagram Protocol (UDP) packets at $25 \mathrm{~Hz}$ to the Front-End Software Architecture (FESA) [19] middleware, where they are received by a dedicated BPM collimator FESA class running on a Front-End Computer (FEC). The beam position measurement is then calculated at $1 \mathrm{~Hz}$ as:

$$
X_{\mathrm{bpm}} \simeq \frac{B}{4} \frac{V_{L}-V_{R}}{V_{L}+V_{R}}
$$

where $V_{L}$ and $V_{R}$ are the induced potentials on the left and right electrodes, and $B$ is the total distance (aperture) between the opposite downstream or upstream BPM electrodes, given by:

$$
B=J_{L}-J_{R}+2 A=G+2 A
$$




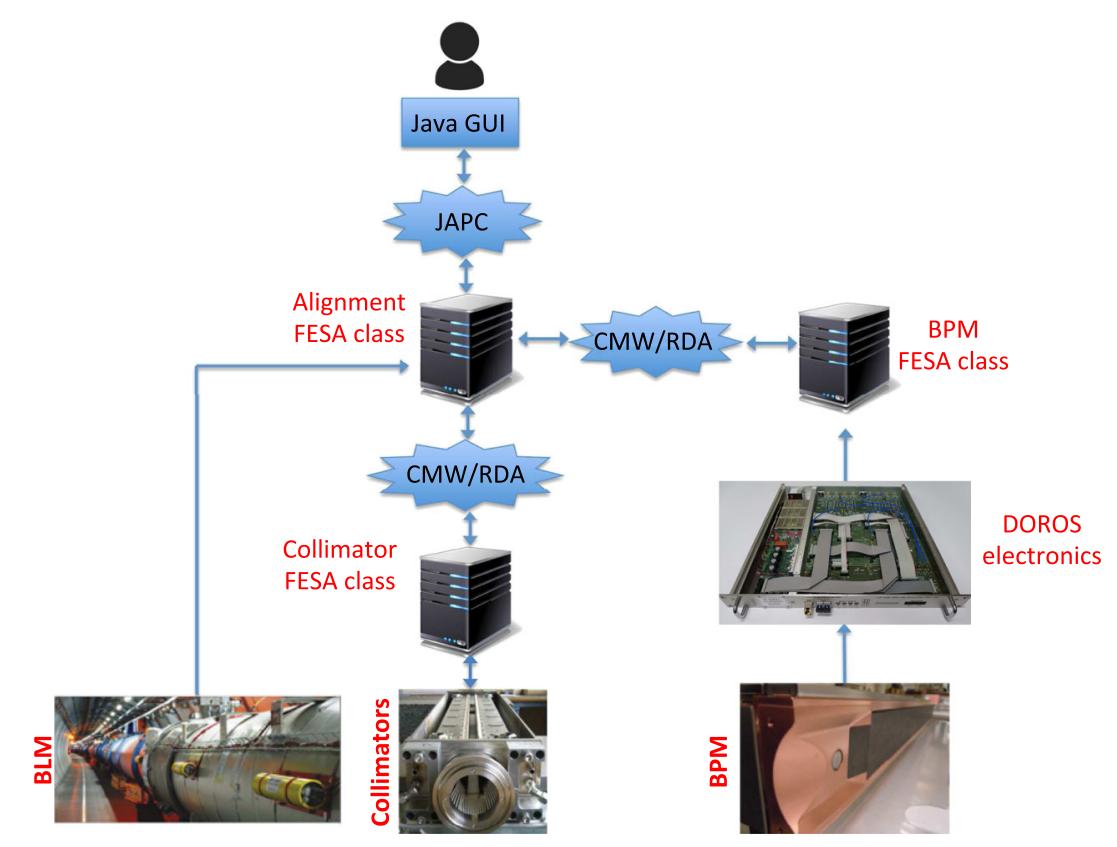

FIG. 2. Software architecture developed for the data acquisition and operation of the embedded collimator BPMs for collimator alignment and beam orbit monitoring.

where $J_{L}$ and $J_{R}$ are the left and right jaw positions in $\mathrm{mm}, G$ is the distance between the graphite surfaces of the opposite jaws, and $A$ is the retraction of the BPM button pick-up with respect to the jaw surface $\left(A_{\text {TCTP }}=8.6 \mathrm{~mm}\right.$, $A_{\text {TCSP }}=8.5 \mathrm{~mm}$ ). The absolute beam position can be calculated as:

$$
X_{\mathrm{abs}}=X_{\mathrm{bpm}}+J_{c}
$$

where $J_{c}=\left(J_{L}+J_{R}\right) / 2$ is the jaw center. The aperture is calculated by the Alignment FESA class from the upstream and downstream jaw positions of the 18 BPM-equipped collimators, which are obtained from the Collimator FESA class [20] through the Common Middleware Remote Device Access (CMW-RDA) framework [21]. It is then sent to the BPM FESA class at $1 \mathrm{~Hz}$. The Alignment FESA class also receives data from all the LHC BLMs (grouped crate by crate in the different IRs) at $100 \mathrm{~Hz}$ via UDP, in order to perform the BLM-based alignment. These BLM signals are also used to stop the BPM-based alignment if they exceed a certain threshold.

The Alignment FESA class was designed to follow specifications of an alignment accuracy of $5 \mu \mathrm{m}$, which is equivalent to the minimum step size of the jaws, within 20 seconds for any jaw gap and beam offset. It also acts as a data concentrator and combines the BLM data for logging purposes. A Java Graphical User Interface (GUI) is used to send alignment commands via the Java API for Parameter Control (JAPC) [22] to the FESA class and visualize the collimator positions, BLM and BPM signals. The overall software architecture showing the different components and their interconnections is presented in Fig. 2. The control system for the embedded BPMs was implemented during LS1 [23], and was first tested using a fully equipped collimator on surface, with a dedicated controls test stand and a stretched wire to simulate the beam, then in SPS with the same prototype collimator used for the initial feasibility studies, and finally in the LHC during the commissioning.

\section{BEAM COMMISSIONING}

\section{A. Calibration of electronics gains and offsets}

Asymmetries in the two electronics channels, which process signals from one pair of BPMs, introduce gains $g$ and offsets $o$ into the measured data [24], which need to be catered for. The corrected electrode signal for a given channel is therefore obtained as:

$$
V_{j}^{\text {corr }}=g_{j} V_{j}+o_{j}
$$

where $j$ denotes the channel number corresponding to a particular jaw corner. These parameters can be determined experimentally by swapping the opposite BPM channels and measuring the signals in the two configurations [13,24], as shown in Fig. 3.

The gain and offset coefficients obtained for each collimator during initial commissioning tests are shown in Table I, and the greater the deviation from unity and zero respectively, the more the gains and offsets result in errors in electrode signals. No values are available for the three collimators not shown in Table I due to issues with the BPM data acquisition. The fact that all values are very close to unity or zero is a clear indication that the asymmetries are small, however they could still introduce errors in the orbit 


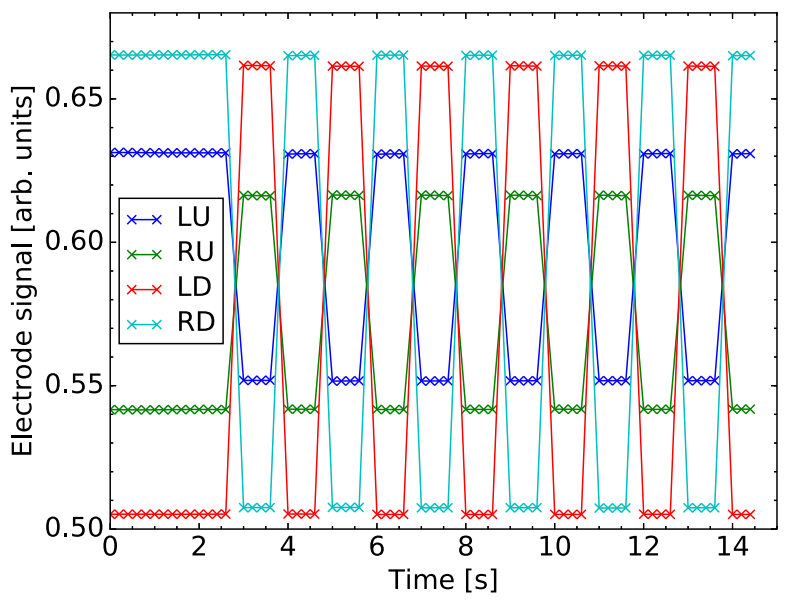

FIG. 3. BPM electrode signals during the electronics calibration test (LU = left-upstream, LD = left-downstream, $\mathrm{RU}=$ right-upstream, RD = right-downstream). The BPM signals are flipped several times in order to obtain repeated measurements to counter for changes in the beam orbit and intensity which affect the electrode signals.

measurements of up to $100 \mu \mathrm{m}$. This set of coefficients is only valid for the BPM apertures and beam intensities at the time of the measurements. Therefore, the DOROS system continuously flips the raw signals at $1 \mathrm{~Hz}$ in order to eliminate any errors at any BPM aperture and beam conditions, and an averaged value is provided by the BPM FESA class.

\section{B. BPM-based collimator alignment}

The BPM-based alignment procedure aims at finding the jaw positions and angles where the beam orbit is centered at both the upstream and downstream sides of the collimators.

TABLE I. Determined gain and offset coefficients to counter for asymmetries in the electronics in the received electrode signals $(\mathrm{UP}=$ upstream, $\mathrm{DW}=$ downstream).

\begin{tabular}{lcccc}
\hline \hline Collimator & Gain UP & Offset UP & Gain DW & Offset DW \\
\hline TCTPH.4L2.B1 & 1.0195 & 0 & 1.0017 & 0 \\
TCTPH.4L5.B1 & 1.0304 & 0.0186 & 0.8891 & 0.0646 \\
TCTPH.4L8.B1 & 1.0106 & -0.0127 & 0.9013 & 0.0592 \\
TCTPV.4L1.B1 & 1.0173 & 0 & 1.0385 & 0 \\
TCTPV.4L2.B1 & 0.9237 & 0.0406 & 0.9462 & 0.0244 \\
TCTPV.4L5.B1 & 0.9930 & 0 & 1.0567 & 0 \\
TCTPV.4L8.B1 & 0.9477 & 0.0304 & 1.1529 & -0.0769 \\
TCTPH.4R1.B2 & 1.0399 & -0.0232 & 1.1634 & -0.1308 \\
TCTPH.4R5.B2 & 0.9151 & 0.0571 & 1.1034 & -0.0240 \\
TCTPH.4R8.B2 & 0.9490 & 0.0223 & 0.9752 & 0.0277 \\
TCTPV.4R1.B2 & 0.9980 & 0 & 1.0089 & 0 \\
TCTPV.4R5.B2 & 1.0089 & 0 & 0.9915 & 0 \\
TCTPV.4R8.B2 & 1.0535 & -0.0340 & 0.9767 & 0.0134 \\
TCSP.A4R6.B1 & 1.0636 & -0.0243 & 0.9906 & 0.0025 \\
TCSP.A4L6.B2 & 0.9216 & 0.0269 & 0.9463 & 0.0230 \\
\hline \hline
\end{tabular}

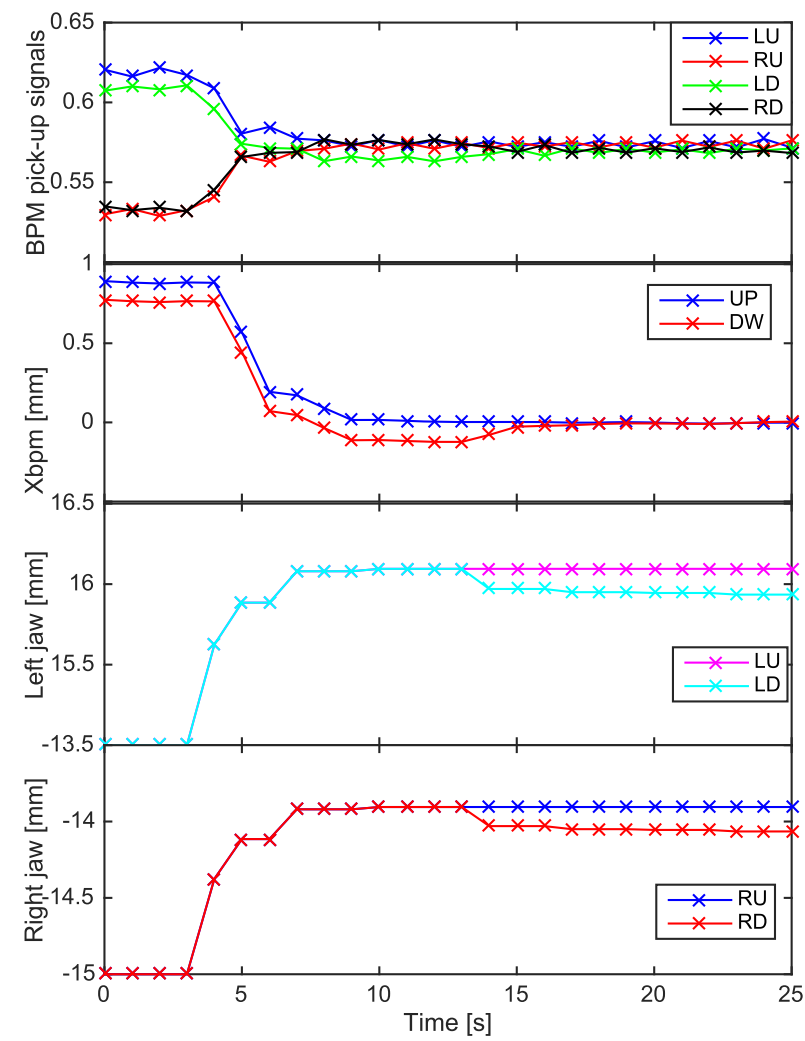

FIG. 4. Example of a BPM-based alignment of the TCSP.A4R6.B1, showing the left-up (LU), left-down (LD), right-up (RU) and right-down (RD) jaw corner positions and electrode signals, as well as the upstream (UP) and downstream (DW) beam positions measured relative to the collimator.

This is achieved by minimizing $X_{\mathrm{bpm}}$ at each pair of opposing BPMs, which is equivalent to finding the jaw positions where $V_{L}=V_{R}$. A successive approximation algorithm was developed and tested in the SPS [13]. It works by moving the left and right jaws in steps, keeping the same gap, until the signals from the opposite upstream electrodes are equalized, and $X_{\mathrm{bpm}}$ is below a tolerance value (e.g. $5 \mu \mathrm{m}$ ). As each jaw corner can be moved independently using a dedicated stepper motor, the alignment algorithm then proceeds to move only the downstream jaw corners until the corresponding signals are also equalized. As a result of non-linearities due to the BPM geometry, the final alignment positions cannot be computed to the required accuracy in one step, and 10-20 steps may be needed until the algorithm converges, taking about $25 \mathrm{~s}$. An example of such an alignment is shown in Fig. 4.

As the BPM-based alignment can be parallelized, the time required to align all BPM collimators is essentially the same as the time required for only one collimator. Therefore, the speed-up obtained when compared to the BLM-based technique is huge, as can be seen in Fig. 5, in which nearly an hour was needed to align only 8 TCTs with the BLM-based technique in 2012, as opposed to $22 \mathrm{sec}-$ onds with the BPM-based technique in 2015. The total 

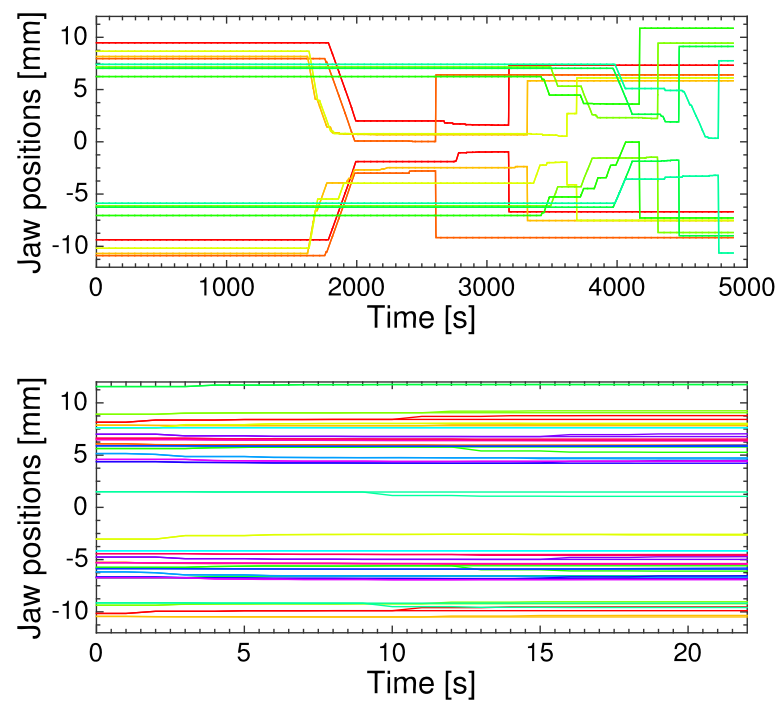

FIG. 5. Time required to align the 8 TCTs in IR1 and IR5 in 2012 with the BLM-based technique (top) and all 16 TCTPs in 2015 with the BPM-based technique (bottom). Each pair of jaws is marked in a separate color.

beam time required for TCT alignment, together with the average time required to align a single TCT collimator over the years, are shown in Fig. 6. There is a marked decrease in time during Run 1 (2010-2013) as the BLM-based technique
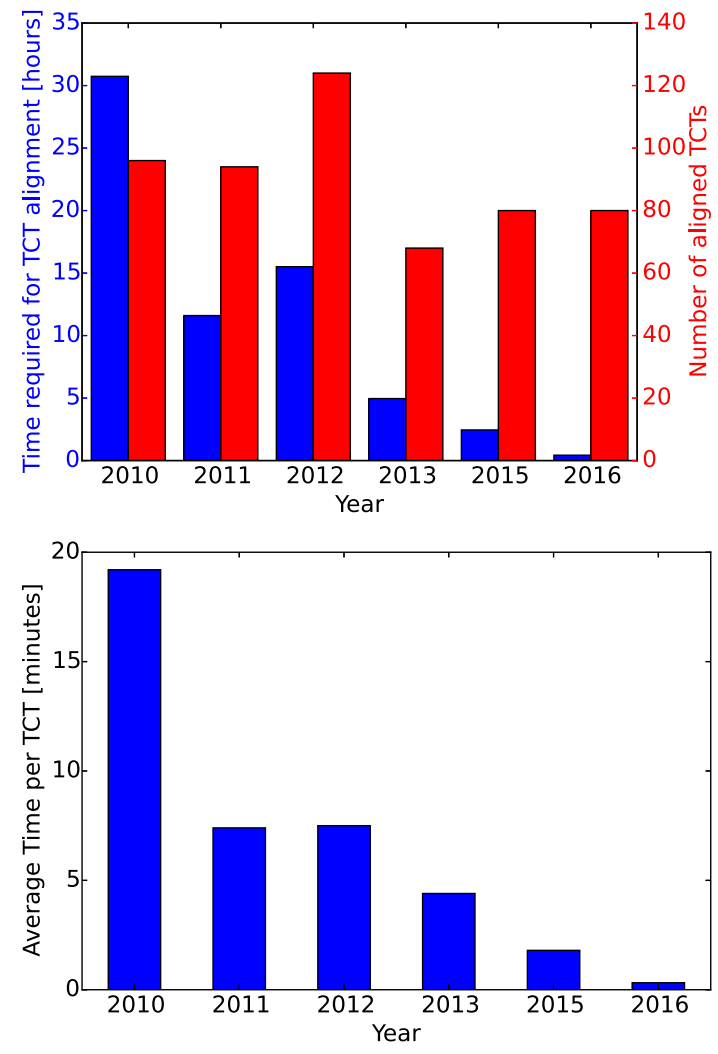

FIG. 6. The total time required for TCT alignment, the number of TCT alignments and the average time required to align a TCT collimator over the years. was automated [5], and then a further decrease in Run 2 (2015-2016) with the introduction of the embedded BPMs, resulting in a total speed-up of a factor 70 for almost the same number of TCT alignments. This is an important achievement considering that the TCTPs are affected by frequent changes in the machine configuration, requiring approximately 100 collimator alignments per year.

During the beam commissioning period at the start of 2016, all 18 BPM collimators were aligned using both the BLM-based and BPM-based techniques at injection and at flat top. As can be seen from the comparison of the beam centers measured shown in Fig. 7, the average of the upstream and downstream BPM-based centers matches the BLM-based center, where the alignment is performed with parallel jaws, within $\pm 150 \mu \mathrm{m}$. The discrepancies in the measurements are due to three factors. First, the BLM-based alignment technique has inherent errors due to the larger jaw step size $(10-20 \mu \mathrm{m})$ required in certain cases (in particular at injection beam energy where the beam size is larger). Second, the jaws are kept parallel with the assumption that the collimator tank is perfectly aligned to the beam, when tilts in the collimator tank with respect to the beam axis can be up to $300 \mu \mathrm{rad}$. In addition, collimators near the experiments are affected by the separation and crossing bumps, which introduce an angle in the closed orbit and may cause a different in measured position between the upstream and downstream part of the collimator of up to $90 \mu \mathrm{m}$.

Tilts in the collimator tank are evident in Fig. 4 (no difference between the upstream and downstream centers is expected for this collimator) and to varying extents in Fig. 7. An alignment of the individual jaw corners to the beam with different jaw tilt angles using the BLM-based technique was used to confirm the presence of collimator tank tilts, as shown in Fig. 8 for three tertiary collimators which presented the largest tilts measured with the BPMbased technique at injection energy. The jaws are deemed to be parallel to the beam, and therefore the error introduced by the tank tilt is corrected, when the minimum jaw gap is achieved after touching the beam on either side with each jaw. Before each measurement, a gentle transverse beam excitation using the transverse damper [25] was done to repopulate the beam halo after the previous alignment, in which some beam is scraped away.

It is also possible to infer the collimator tank tilt by considering the difference of the beam size measured using the BLM-based alignment technique when compared to the nominal beam size [5]. The misalignment angle $\alpha$ is estimated to be

$$
\alpha \simeq \frac{2 n_{1} \Delta \sigma}{L},
$$

where $\Delta \sigma$ is the difference between the real and inferred beam size at the collimator, $n_{1}$ is the cut of a reference collimator in unit $\sigma$ and $L$ is the length of the collimator jaw. For example, for a reference collimator cut of $3 \sigma$, a real $1 \sigma$ 


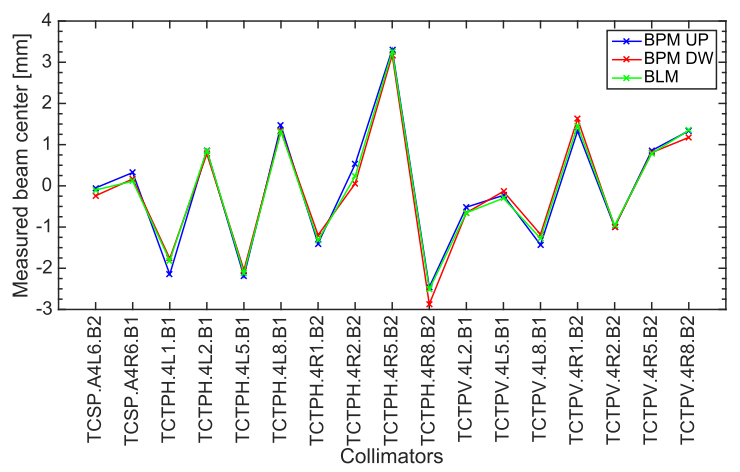

(a)Absolute BPM and BLM centers at injection

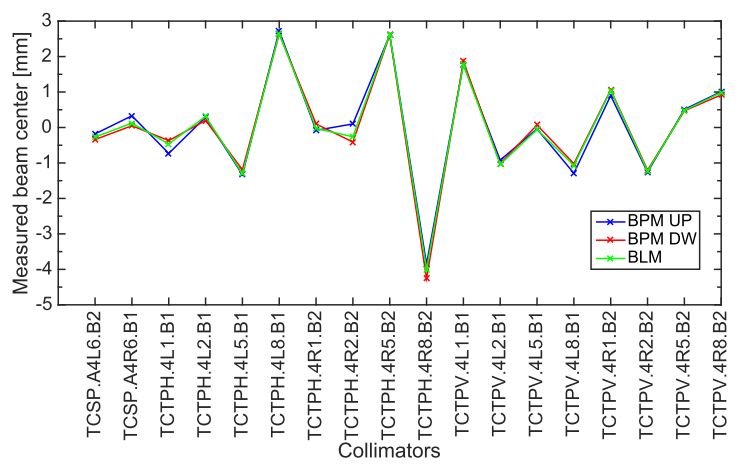

(b)Absolute BPM and BLM centers at flat top

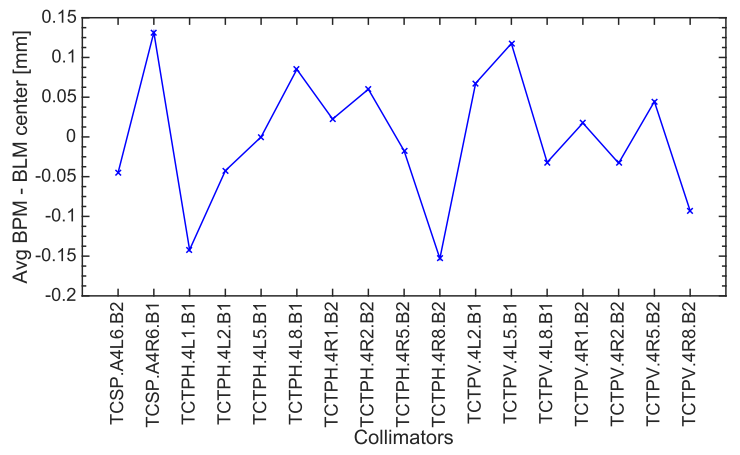

(c)BPM-based vs BLM-based centers at injection

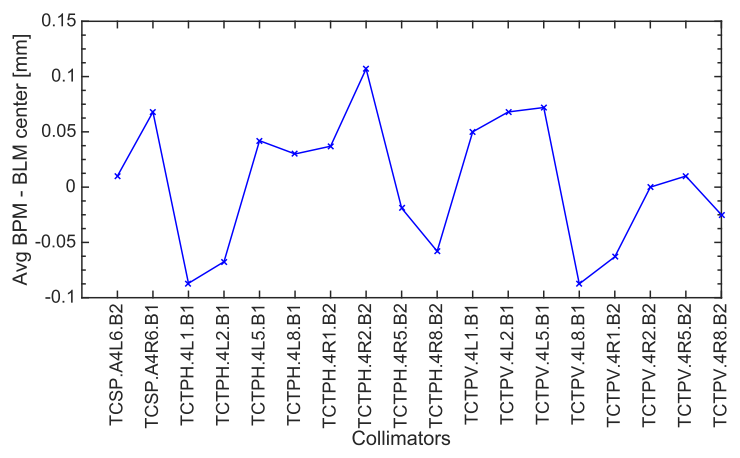

(d)BPM-based vs BLM-based centers at flat top

FIG. 7. A comparison of the absolute beam centers measured using the BLM-based and BPM-based techniques, and the differences between the average of the upstream and downstream BPM-based positions and the BLM-based positions, at injection and flat top.

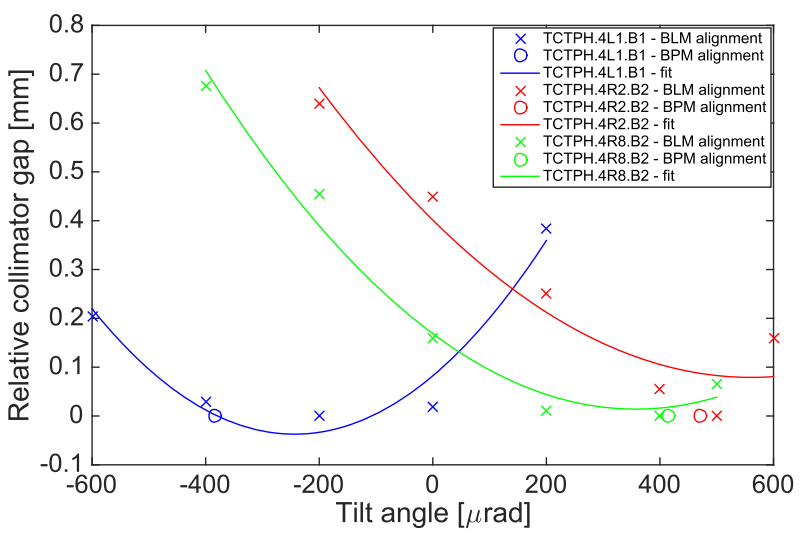

FIG. 8. Measurements of tilts in three collimators from BPMbased and BLM-based alignment.

beam size of $200 \mu \mathrm{m}$, an inferred $1 \sigma$ beam size of $250 \mu \mathrm{m}$ and a typical collimator jaw length of $1 \mathrm{~m}$, the calculated angle is $400 \mu \mathrm{rad}$. Note that aligning the collimator at a tighter reference cut would not imply a smaller $\alpha$; rather, the inferred beam size would be larger [5]. The computed values of $\alpha$ are also reported in Fig. 8. A comparison of the expected tilts obtained with MADX and the measurements for the same collimators is shown in Fig. 9, where it is evident that the expected changes in the closed orbit are not enough to explain the observed differences between the upstream and downstream readings.

The operational settings depend not only on the measured beam center, but also on the beam size at the collimator, which cannot be measured using the BPM-based technique. From experience during LHC operation in Run 1, the determination of the beam size using the BLM-based alignment technique provides a consistent collimation hierarchy at injection, but not at top energy [26]. This is because the collimator gaps are smaller in $\mathrm{mm}$ at top energy, which makes the alignment procedure more sensitive to gap measurement errors. The top energy collimator settings

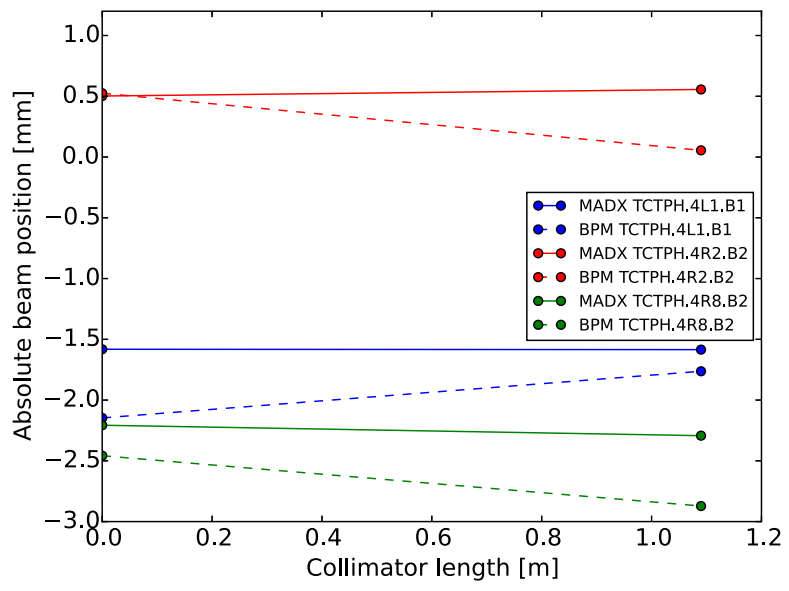

FIG. 9. Comparison of upstream and downstream beam positions in three collimators expected from MADX and those found in the BPM-based alignment. 
therefore rely on the nominal betatron beam size, instead of the inferred beam size. Therefore, the BLM-based technique is currently used during the yearly alignment at injection energy, while following the excellent commissioning results presented in this paper, the BPM-based technique is now routinely used for the alignment of collimators with embedded BPMs during the initial commissioning at top energy, and for the more frequent changes in the machine configuration at this energy throughout the year.

\section{BPM nonlinearity corrections}

As derived in [13], Eq. (1) holds if the collimator geometry is a perfect cylinder. However, the two jaws form parallel plates, which introduce nonlinearities in the measurements for large beam offsets and BPM apertures. Detailed simulations were already performed for the prototype collimator, and were reported in $[13,27]$. In order to correct for these non-linearities, orbit measurements must be performed at a variety of operational gaps and offsets. A 2D $m n$-degree polynomial with coefficients $c_{p q}$ can then be fitted to the data:

$$
X_{\mathrm{bpm}}=\sum_{p, q=0}^{m, n} c_{p q} X_{\mathrm{raw}}^{p} B^{q},
$$

where $X_{\text {raw }}$ is the difference-over-sum of the opposite electrode signals and therefore excludes the BPM aperture as a parameter.

The measurements were done via collimator scans, using a dedicated Java GUI application, which takes as input the starting and stopping gap, a margin with respect to the center (to avoid scraping the beam), a time interval between each step, and step sizes for the beam offset and the jaw gap. Initially, a $4 \sigma$ cut was made with the horizontal and vertical TCPs in both beams, to ensure that a large range could be probed. The scans were performed from a gap of $30 \mathrm{~mm}$ (maximum outer positions) down to $8 \sigma$, as well as at offsets in the range of $\pm 10 \mathrm{~mm}$, and took around 1.5 hours to perform for all collimators in parallel. The scans were done at injection and top energy. The nonlinear relationship between $X_{\text {beam }}$ (the beam offset) and the measured beam position relative to the collimator center $X_{\mathrm{bpm}}$ for the TCTPV.4R2.B2 is shown in Fig. 10.

The linearity factor parameter $L_{f}[13]$ is used as a nonlinear conversion coefficient between the linearized and original beam positions:

$$
L_{f}=\frac{X_{\mathrm{bpm}}}{X_{\text {beam }}}
$$

and can be used to further illustrate the BPM nonlinearity with aperture and beam offset, as shown in Fig. 11. Similarly as in [13], the parameters of the 2D fit were set to $m=5$ and $n=3$ (therefore referred to as Poly53), and an example of a fit applied to the measurements for the same collimator is shown in Fig. 12. Contrary to what was

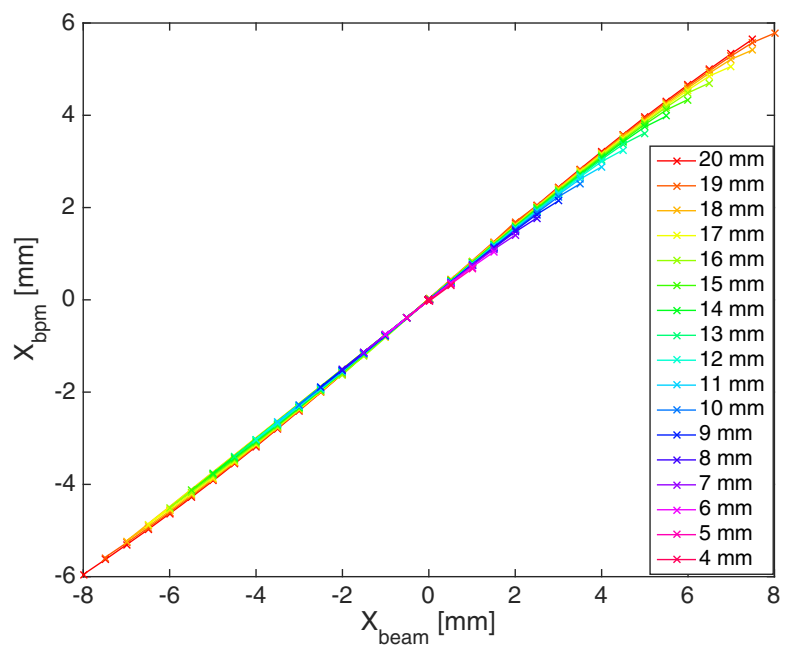

FIG. 10. Nonlinear relationship between $X_{\text {beam }}$ and $X_{\mathrm{bpm}}$ for TCTPV.4R2.B2, depending on the beam offset and jaw gap (see legend).

observed in the simulations, all coefficients of the Poly53 fit needed to be kept in order to ensure $R^{2}=1$. The $2 \mathrm{D}$ fit coefficients obtained for all 36 collimator BPMs during collimator scans at flat top are summarized in Fig. 13. Similar results were obtained from the collimator scans done at injection, although as the beam size is larger at lower energy, the scan range was more restricted than at flat top in $\mathrm{mm}$. The resulting plot of the beam position corrected using the fit coefficients $X_{\text {corr }}$ as a function of $X_{\text {beam }}$ is shown in Fig. 14.

\section{Effects of beam offsets in the orthogonal plane}

The collimator BPMs are positioned to measure the beam offset in the collimator plane (horizontal or vertical). Due to the geometry, beam offsets in the orthogonal plane may

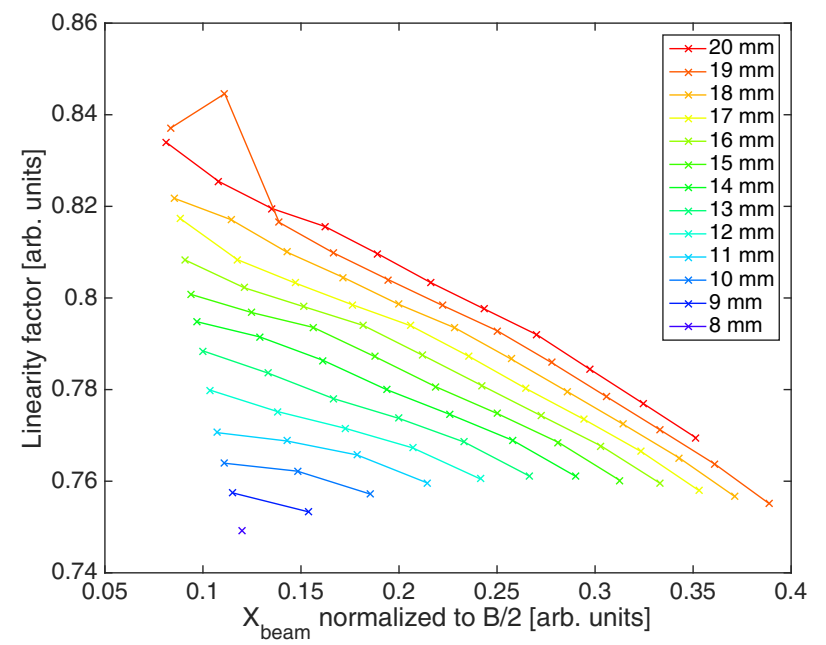

FIG. 11. Linearity factor as a function of the beam offset normalized to the BPM aperture for the TCTPV.4R2.B2 for different jaw gaps (see legend). 


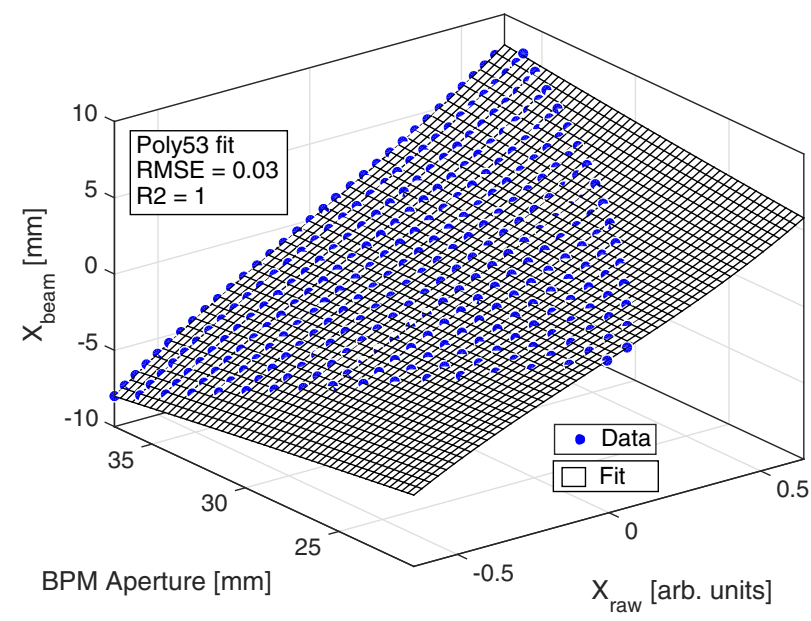

FIG. 12. The Poly53 2D polynomial fit used to correct the nonlinear BPM readings of the TCTPV.4R2.B2 for the operational jaw motion range.

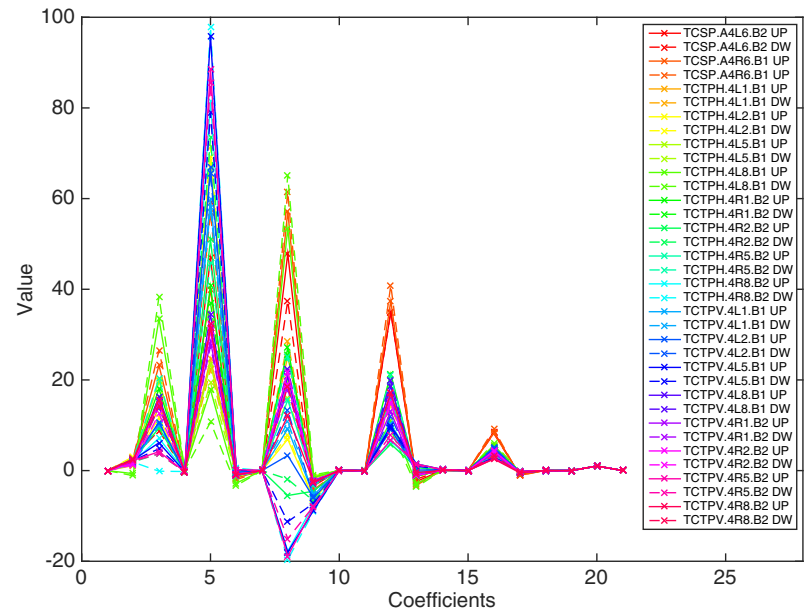

FIG. 13. The 2D fit coefficients $c_{p q}$ obtained for all 36 collimator BPMs during the collimator scans at flat top.

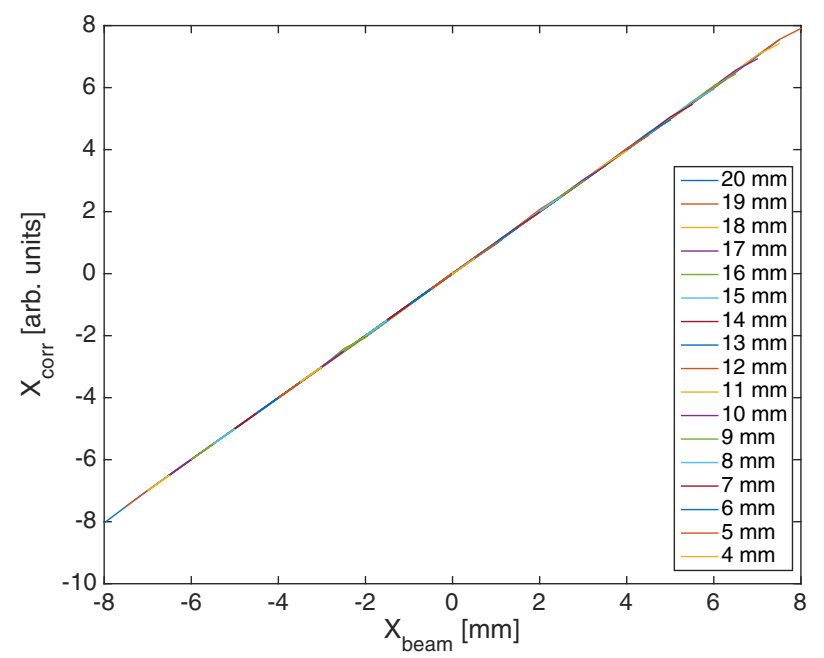

FIG. 14. The resulting corrected plot of $X_{\text {corr }}$ as a function of $X_{\text {beam }}$. affect the measurements. Therefore, tests were conducted to verify the extent of these effects, which could be up to several $\mathrm{mm}$ due to the crossing and separation orbit bumps placed at the experimental points in order to bring the beams into collisions. Orbit bumps in the plane orthogonal to the collimation plane were placed in the TCTPH.4L5.B1, TCTPV.4L5.B1, and TCSP.A4R6.B1. A range of $\pm 4 \mathrm{~mm}$

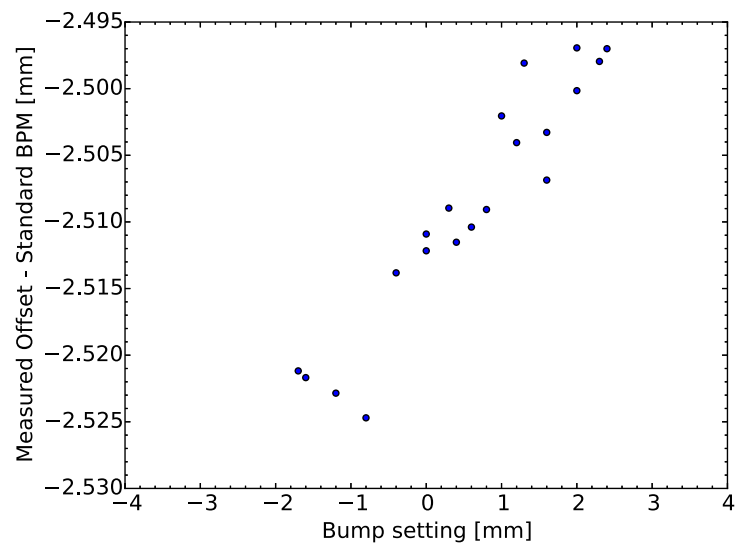

(a)Vertical bump in TCTPH.4L5.B1

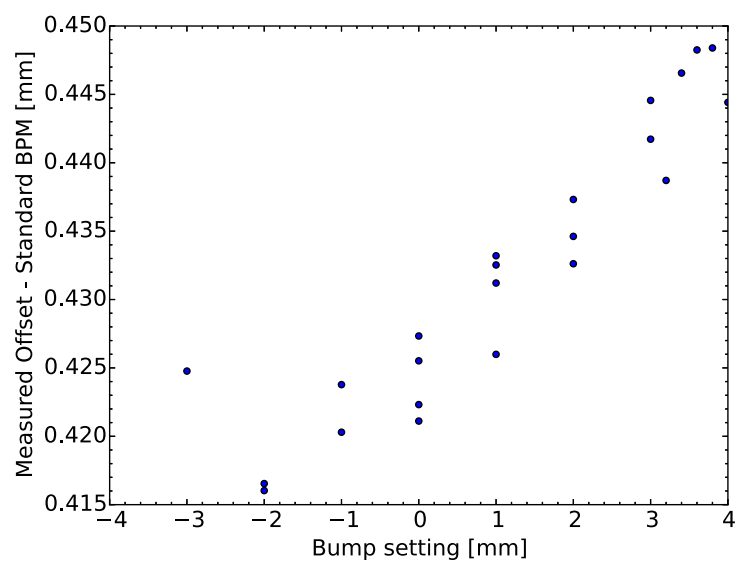

(b)Horizontal bump in TCTPV.4L5.B1

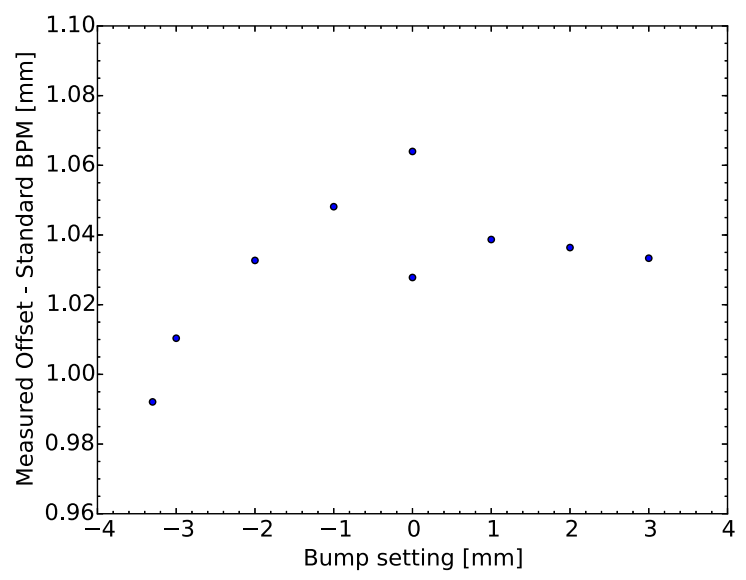

(c)Vertical bump in TCSP.A4R6.B1

FIG. 15. Estimated change in $X_{\mathrm{abs}}$ for various orbit bumps in the orthogonal plane of three BPM collimators. 
was used where possible. Bumps with an angle between upstream and downstream larger than $10 \mu \mathrm{rad}$ were not possible without risking to touch the aperture. There are different offsets for the nominal orbit (bump setting set to $0 \mathrm{~mm}$ ) as the collimator was realigned at the start of each scan in the negative and positive bump directions.
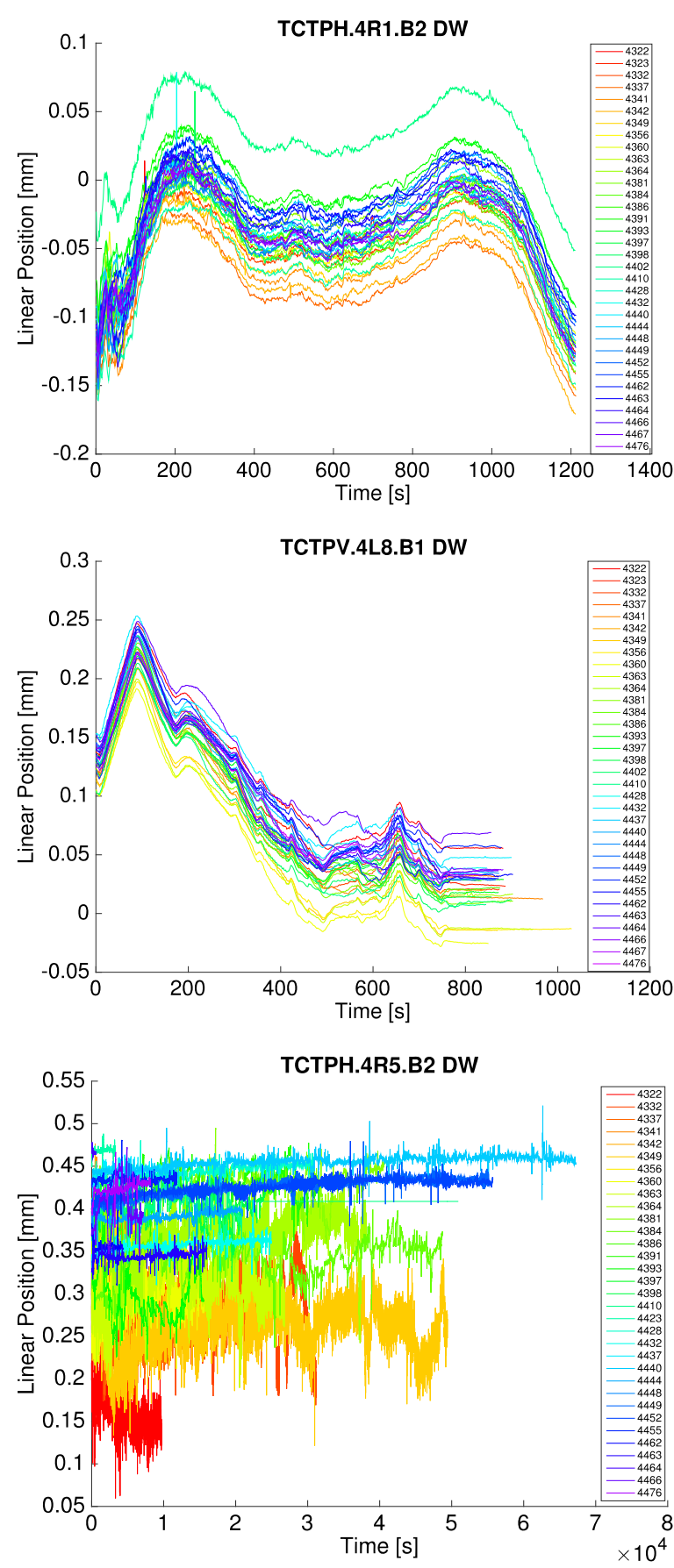

FIG. 16. Beam positions measured relative to the centers of selected collimators during the ramp (top), squeeze (center), and stable beams (bottom) in 2015, before they were used in the collimator settings generation. Each number in the legend corresponds to a separate fill for physics.
Plots of the measured offset in the collimator BPMs relative to a standard BPM in the same plane a few metres away are shown in Fig. 15. The change in the orbit (less than $15 \mu \mathrm{m}$ from a zero bump setting) measured by the collimator BPMs at the TCTPs was deemed too small during the test to be cross-checked with the BLM-based technique. However, a larger shift (70 $\mu \mathrm{m}$ with respect to the orbit at the zero bump setting) measured at the TCSP was found to be present also with BLM-based alignment. Therefore, the conclusion is that the orbit change in the collimator plane was real and not imaginary due to nonlinearities coming from the BPM geometry, and shifts of the beam orbit in a reasonable range in the plane orthogonal to the collimator BPMs should not affect the readings. This is particularly important should the collimator 5th axis need to be deployed, and therefore move the collimator in the plane orthogonal to the collimation plane, to present a fresh collimating surface to the beam following an accident scenario such as an asynchronous dump [28].

\section{PERFORMANCE IN STANDARD OPERATION}

\section{A. Fill-to-fill performance}

A fill-to-fill analysis was performed for the collimator BPM data acquired during several parts of the machine cycle in the standard p-p run in 2015, as shown in Fig. 16 for several TCTPs in different IRs, and for different parts of the machine cycle, i.e., ramp, squeeze, and stable beams. None of the curves start from a zero beam position relative the collimator center, as during 2015 the collimator settings were based on measurements from the BLM-based alignment. A good fill-to-fill reproducibility is noted, which was exploited in the dynamic parts of the cycle (ramp and squeeze) by means of a feed-forward into the collimator functions as explained in the following section.

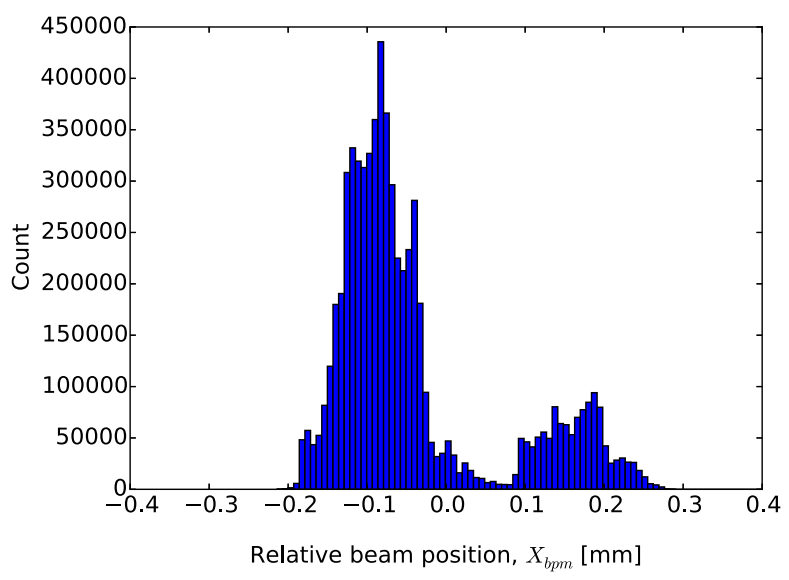

FIG. 17. The distribution of the measured orbit at the TCTPH.4L1.B1 during all proton-proton stable beams periods in 2016. $99 \%$ of the orbit drifts with respect to the collimator center are within $235 \mu \mathrm{m}$. 


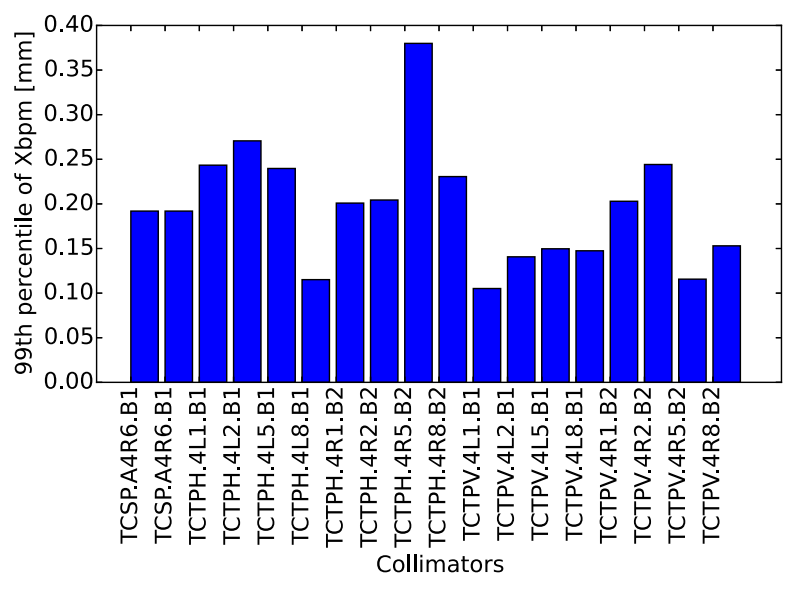

FIG. 18. Distribution of the $99 \%$ of the all orbit drifts at each of the BPM collimators in stable beams.

The good fill-to-fill reproducibility is also evident in the distributions of the measured orbits (see example in Fig. 17), which were computed for all collimators in order to determine the values corresponding to $99 \%$ of all orbit
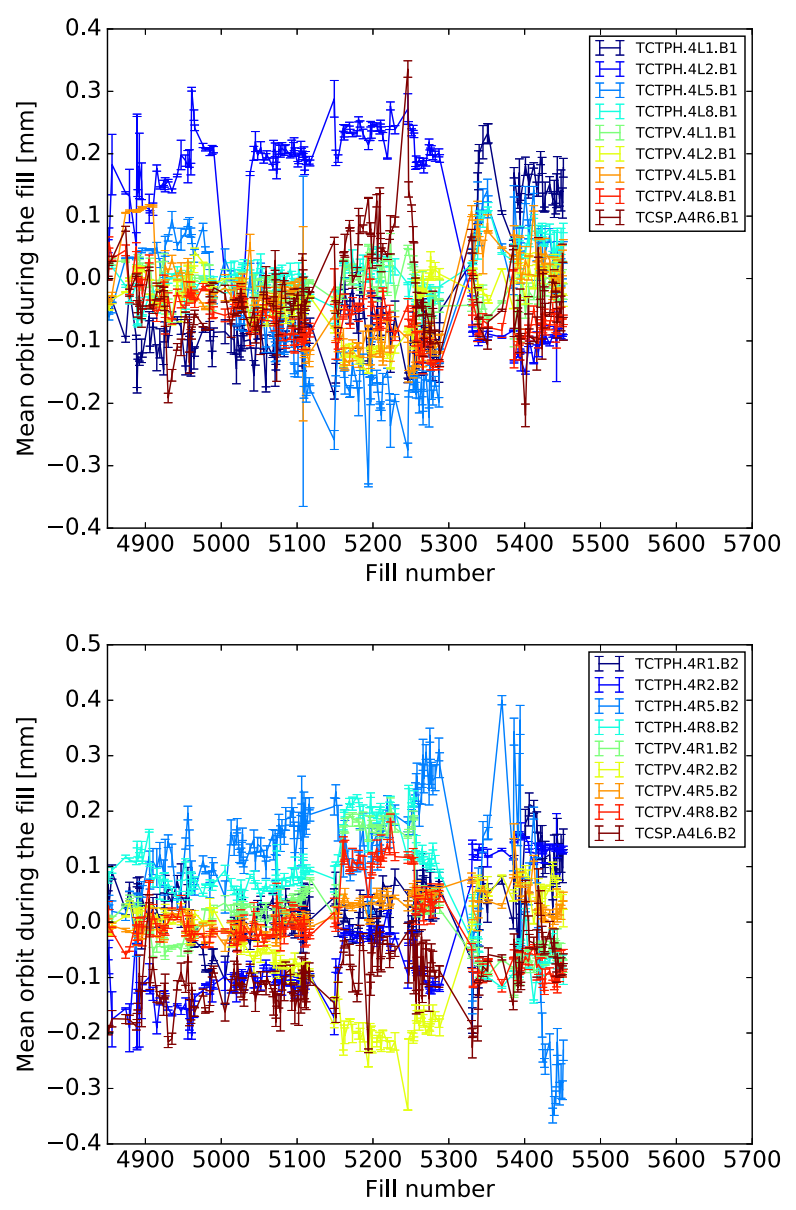

FIG. 19. The average beam positions measured during the stable beams period each proton physics fill in 2016, together with the standard deviation as the error bar, for B1 (top) and B2 (bottom) collimators. drifts, as shown in Fig. 18. The average beam positions measured during the stable beams period for each of the 155 proton physics fills in 2016, together with the standard deviation as the error bar, is reported in Fig. 19. Small shifts are observed between before and after the week-long technical stops, which correspond to periods with a break in the fill number along the $x$-axis. In general, however, the shifts are remarkably small, and both the inter-fill and intrafill stability are very good. The average of the upstream and downstream beam positions measured at each pair of neighboring horizontal and vertical TCTPs in the same beam and side of each IR, averaged over the entire stable beams duration of each of the proton physics fills in 2016, are plotted in Fig. 20 to provide another view of the orbit stability.

\section{B. Generation of collimator settings}

Up to 2015, the collimator settings during dynamic parts of the cycle, such as the ramp and adjust beam modes, were
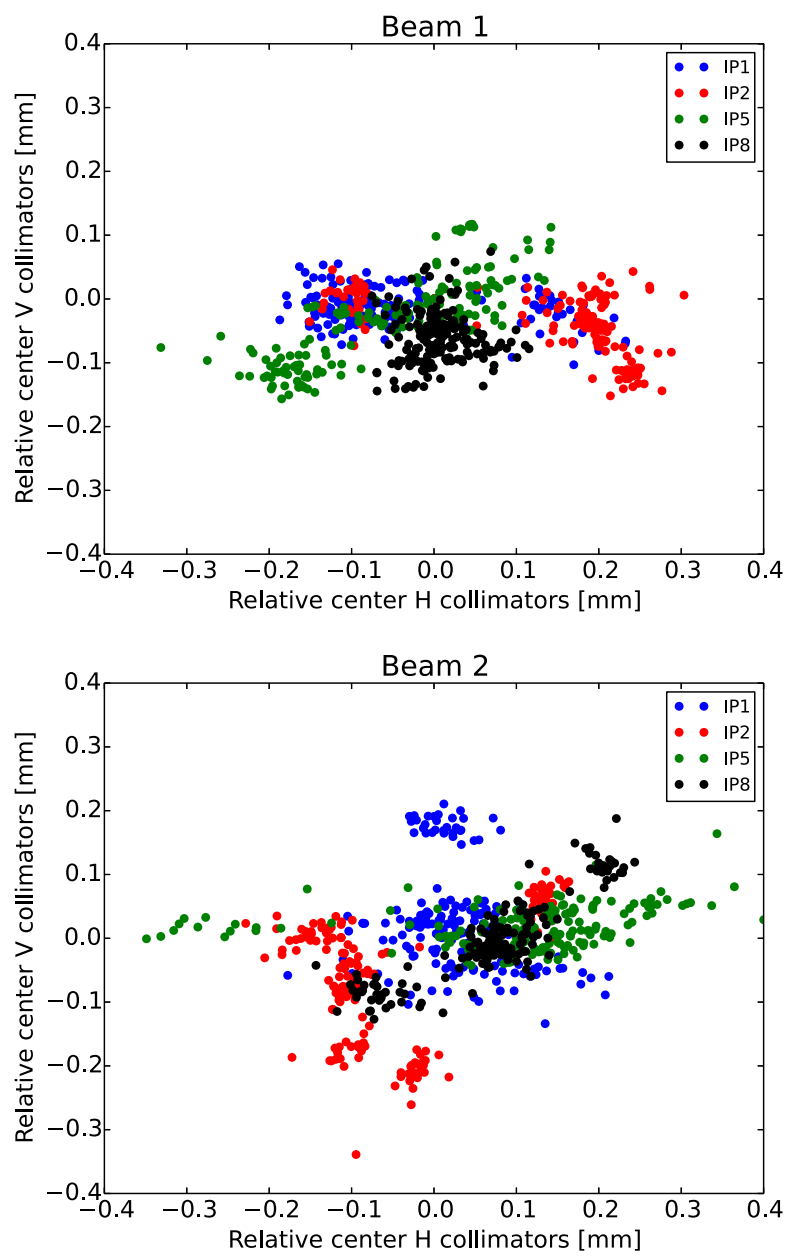

FIG. 20. Average of upstream and downstream beam positions measured at each pair of neighboring horizontal and vertical TCTPs in B1 (top) and B2 (bottom), averaged over the entire stable beams duration of each proton physics fill in 2016. 


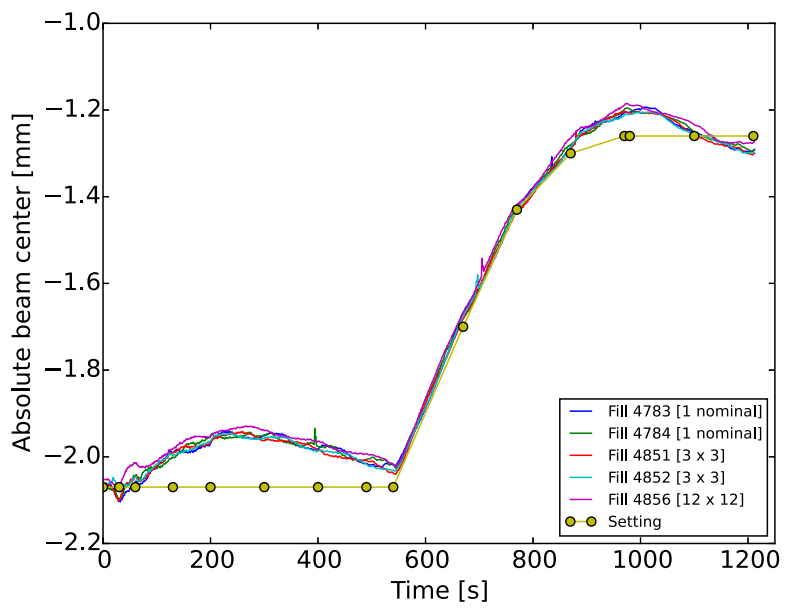

FIG. 21. The absolute measured beam orbit at the collimator together with the setting for the TCTPH.4L5.B1 during the combined ramp and squeeze.

generated assuming a linear interpolation of the beam center from the value measured during beam-based alignment at the start (e.g. start of ramp) to the value measured at the end (e.g. flat top) [29]. During the squeeze, a simulated orbit as a function of time was used, starting from the beambased alignment centers measured at flat top and at the end of the squeeze as start and end points respectively. In addition, the jaw positions are interlocked at all times by inner and outer limits for each jaw corner and gaps, as well as redundant $\beta^{*}$ and energy limits on the gap [30].

As mentioned previously, a good fill-to-fill reproducibility was noted in the beam position measurements. In addition, the dynamic behavior of the measured orbit during each fill corresponded to the predicted beam position as expected from simulations done using MADX [31]. Therefore, for 2016 all functions for the BPM collimator settings were generated by scaling the predicted beam position from MADX to the start and end point measured using BPM-based alignment. An example is shown in Fig. 21, where the beam center in the ramp beam process is shown together with measurements from subsequent fills.

\section{Beam interlocks}

The direct monitoring of the orbit at the TCSPs and TCTPs would have to be interlocked if the orbit margins in the collimator settings are going to be reduced to push the $\beta^{*}[32,33]$. This means that the beams would be preventively dumped by the software interlock system (SIS) [34] to avoid dangerous conditions for the LHC, if the measured orbit exceeds a predefined safe interval. An interlock threshold scan was performed to determine the number of dumps that would have occurred during each stage of the machine cycle: ramp, squeeze, adjust, and stable beams, as a function of possible thresholds.

A data set of 155 fills, comprising all standard proton physics fills in 2016, was used. A dump is registered if both
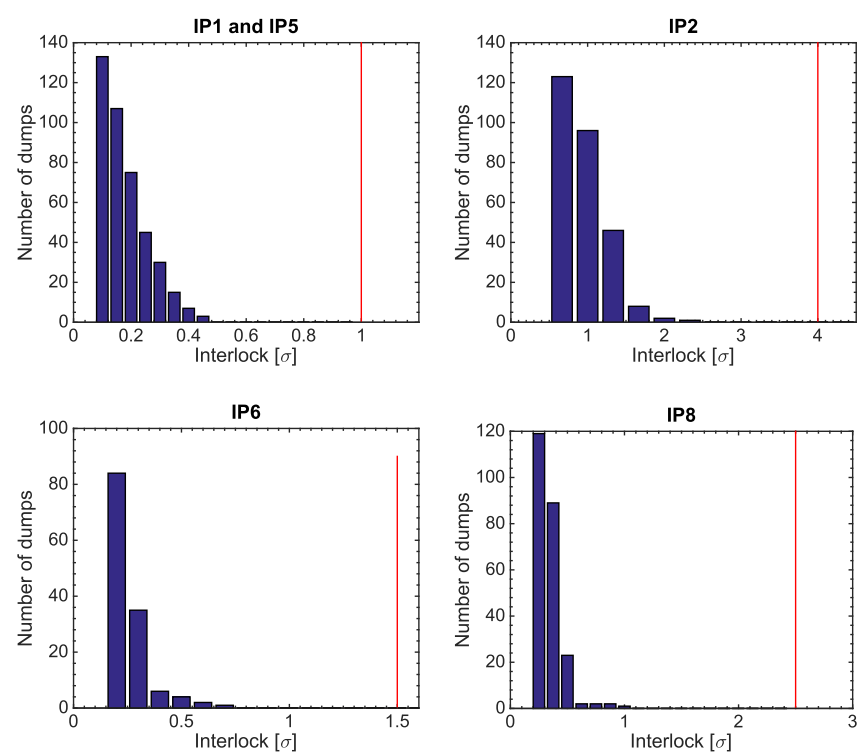

FIG. 22. Number of predicted dumps during stable beams for different BPM interlock thresholds for TCTP and TCSP collimators separated by IR configuration in nominal beam $\sigma$. The red line indicates the proposed threshold for each configuration.

the upstream and downstream relative beam positions exceed the threshold at any collimator. The results for stable beams are shown in Fig. 22, and similar results were obtained for other parts of the machine cycle. Based on this analysis, conservative interlock limits which would result in an improvement compared to previously assumed margins are shown in Table II for operation with squeezed beams. The values were selected to be twice the largest recorded offset. The interlock value at all other times would be $4 \sigma$, which would be reduced to the values shown in Table II during the squeeze. These interlocks could be potentially tightened further after accumulating more experience, without risking spurious dumps which would result the loss in machine availability outweighing the gain from the increase in luminosity. Assuming tighter collimator settings and beam-beam separation, a $\beta^{*}$ of $30-35 \mathrm{~cm}$ is within reach [9], and the availability of BPM interlocks will ensure that the tighter orbit margins are never violated. This is particularly important if the phase advance from the dump kicker to the TCT is not perfect.

TABLE II. Proposed beam orbit interlock thresholds for the collimator BPMs for operation at $6.5 \mathrm{TeV}$ with squeezed beams $\left(\beta^{*}=40 \mathrm{~cm}\right)$.

\begin{tabular}{lcc}
\hline \hline IR Configuration & Interlock value $[\sigma]$ & Interlock value [mm] \\
\hline IR1 and IR5 & 1 & $\mathrm{H}=1.050, \mathrm{~V}=0.660$ \\
IR2 & 4 & $\mathrm{H}=0.615, \mathrm{~V}=0.615$ \\
IR6 & 1.5 & $\mathrm{H}=0.770$ \\
IR8 & 2.5 & $\mathrm{H}=0.875, \mathrm{~V}=0.980$ \\
\hline
\end{tabular}




\section{CONCLUSIONS}

Beam position monitors embedded in movable collimator jaws can provide a direct measurement of the beam orbit, which can be used to center the jaws around the beam. A first upgrade of the LHC collimation system took place in LS1, when 18 collimators with this new feature were installed. A successful commissioning campaign was carried out to ensure the correct functionality of the BPMs and of the control software, as well as correct any nonlinearities present. The goal of significantly reducing the time to realign the collimators for several frequent changes of machine configurations was achieved by a factor 70 . BPM-based alignment is now confirmed as the standard technique for these collimators. The fill-to-fill analysis of orbit measurements during the LHC machine cycle demonstrates the quality and reliability of the system, and together with the good reproducibility of the orbit and its dynamic behavior during the ramp and squeeze, indicate that it would be possible to deploy beam interlocks in order to reduce the existing collimation hierarchy margins which account for orbit drifts. In addition, the orbit measured at the collimators has been used to update the collimator functions to ensure that the collimators are well-centered around the beam even during dynamical phases of the cycle. The successful deployment and usage of the embedded BPMs augurs well for future upgrades of the LHC collimation system.

\section{ACKNOWLEDGMENTS}

The authors would like to thank the various equipment groups (BE/ABP, BE/BI, EN/STI, TE/VSC) who helped out with the surface tests, hardware commissioning and installation of the BPM collimators, as well as the LHC operational crew (BE/OP) for support during the beam measurements performed.

[1] O. S. Brüning, P. Collier, P. Lebrun, S. Myers, R. Ostojic, J. Poole, and P. Proudlock, Report No. CERN-2004-003V1, 2004.

[2] R. W. Aßmann et al., in Proceedings of the 10th European Particle Accelerator Conference, Edinburgh, Scotland, 2006 (EPS-AG, Edinburgh, Scotland, 2006), p. 1538.

[3] D. Wollmann et al., in Proceedings of the International Particle Accelerator Conference, Kyoto, Japan (ICR, Kyoto, 2010), p. 1237.

[4] B. Dehning et al., in Proceedings of the 22nd Particle Accelerator Conference, PAC-2007, Albuquerque, NM (IEEE, New York, 2007), p. 4192.

[5] G. Valentino, R. W. Assmann, R. Bruce, S. Redaelli, A. Rossi, N. Sammut, and D. Wollmann, Semiautomatic beam-based LHC collimator alignment, Phys. Rev. ST Accel. Beams 15, 051002 (2012).

[6] G. Valentino, R. W. Assmann, R. Bruce, S. Jackson, S. Redaelli, B. Salvachua, N. Sammut, D. Wollmann, and
C. Zamantzas, in Proceedings of ICALEPCS, San Francisco, California, USA, 2013 (Jacow, San Francisco, 2013), p. 1430.

[7] G. Valentino, Report No. CERN-THESIS-2013-208, 2013.

[8] R. Bruce, R. W. Assmann, and S. Redaelli, Calculations of safe collimator settings and $\beta^{*}$ at the CERN Large Hadron Collider, Phys. Rev. ST Accel. Beams 18, 061001 (2015).

[9] R. Bruce, C. Bracco, R. D. Maria, M. Giovannozzi, A. Mereghetti, D. Mirarchi, S. Redaelli, E. Quaranta, and B. Salvachua, Reaching record-low $\beta^{*}$ at the CERN Large Hadron Collider using a novel scheme of collimator settings and optics, Nucl. Instrum. Methods Phys. Res., Sect. A 848, 19 (2017).

[10] D. Wollmann, O. Aberle, R. W. Assmann, A. Bertarelli, C. Boccard, R. Bruce, F. Burkart, M. Cauchi, A. Dallocchio, D. Deboy, M. Gasior, R. Jones, S. Redaelli, A. Rossi, and G. Valentino, in Proceedings of the 2nd International Particle Accelerator Conference, San Sebastiáán, Spain (EPS-AG, Spain, 2011), p. 3747.

[11] D. Wollmann, A. A. Nosych, G. Valentino, O. Aberle, R. W. Assmann, A. Bertarelli, C. Boccard, R. Bruce, F. Burkart, E. Calvo, M. Cauchi, A. Dallocchio, D. Deboy, M. Gasior, R. Jones, S. Redaelli, and A. Rossi, Beam feasibility study of a collimator with in-jaw beam position monitors, Nucl. Instrum. Methods Phys. Res., Sect. A 768, 62 (2014).

[12] G. Valentino, R. W. Assmann, R. Bruce, M. Gasior, D. Mirarchi, A. Nosych, S. Redaelli, B. Salvachua, and N. Sammut, in Proceedings of the 4th International Particle Accelerator Conference, IPAC-2013, Shanghai, China, 2013 (JACoW, Shanghai, China, 2013), p. 3439.

[13] G. Valentino, A. A. Nosych, R. Bruce, M. Gasior, D. Mirarchi, S. Redaelli, B. Salvachua, and D. Wollmann, Successive approximation algorithm for beam-positionmonitor-based LHC collimator alignment, Phys. Rev. ST Accel. Beams 17, 021005 (2014).

[14] B. Salvachua et al., in Proceedings of the 5th LHC Beam Workshop, Evian, France, 2014 (CERN, Evian, 2014), p. 111.

[15] A. Dallocchio, A. Bertarelli, C. Boccard, F. Carra, M. Gasior, L. Gentini, and M. Timmins, in Proceedings of the 2nd International Particle Accelerator Conference, San Sebastiáán, Spain (EPS-AG, Spain, 2011), p. 1611.

[16] M. Gasior, J. Olexa, G. Baud, and G. Valentino, in Proceedings of IBIC, Barcelona, Spain, 2016 (Jacow, Barcelona, 2016).

[17] M. Gasior, J. Olexa, and R. J. Steinhagen, in Proceedings of BIW'12 Workshop, Newport News, VA, USA, 2012 (Jacow, Newport News, 2012).

[18] M. Gasior, J. Olexa, and R. J. Steinhagen, in Proceedings of DIPAC'11, Hamburg, Germany, 2011 (Jacow, Hamburg, 2011), p. 98.

[19] M. Arruat et al., in Proceedings of ICALEPCS, Knoxville, Tennessee, USA, 2007 (Jacow, Knoxville, 2007), p. 310.

[20] A. Masi and R. Losito, LHC collimators low level control system, IEEE Trans. Nucl. Sci. 55, 333 (2008).

[21] K. Kostro, V. Baggiolini, F. Calderini, F. Chevrier, S. Jensen, R. Swoboda, and N. Trofimov, in Proceedings of the 8th European Particle Accelerator Conference, Paris, 2002 (EPS-IGA and CERN, Geneva, 2002), p. 2028. 
[22] V. Baggiolini et al., in Proceedings of ICALEPCS, Geneva, Switzerland, 2005 (Jacow, Geneva, 2005).

[23] G. Valentino, G. Baud, M. Gasior, S. Jackson, L. Jensen, J. Olexa, S. Redaelli, and J. Wenninger, in Proceedings of ICALEPCS, Melbourne, Australia, 2016 (Jacow, Melbourne, 2016), p. 306.

[24] M. Gasior, in Proceedings of IBIC, Oxford, UK, 2013, p. 85.

[25] W. Höfle, R. W. Assmann, S. Redaelli, R. Schmidt, D. Valuch, D. Wollmann, and M. Zerlauth, in Proceedings of the 3rd International Particle Accelerator Conference, New Orleans, LA, 2012 (IEEE, Piscataway, NJ, 2012), p. 4059.

[26] S. Redaelli, R. W. Aßmann, R. Bruce, A. Rossi, and D. Wollmann, in Proceedings of HB2010, Morschach, Switzerland, 2010 (Jacow, Morschach, 2010), pp. 395-399.

[27] A. Nosych, C. Boccard, and M. Gasior, in Proceedings of DIPAC'11, Hamburg, Germany, 2011 (Jacow, Hamburg, 2011), p. 71.

[28] A. Bertarelli, R. Bruce, F. Carra, A. Dallocchio, M. Guinchard, N. Mariani, L. Lari, S. Redaelli, and A. Rossi, in Proceedings of the Machine Protection Panel Workshop, Annecy, France, 2013 (CERN, Annecy, 2013), pp. 108-112.

[29] R. Bruce, R. W. Aßmann, and S. Redaelli, in Proceedings of the 2nd International Particle Accelerator Conference, San Sebastiáán, Spain (EPS-AG, Spain, 2011), p. 3753.

[30] G. Valentino, R. Bruce, and S. Redaelli, in Proceedings of the Machine Protection Panel Workshop, Annecy, France, 2013 (CERN, Annecy, 2013), pp. 87-92.

[31] H. Grote and F. Schmidt, in Proceedings of the 2003 Particle Accelerator Conference, Portland, OR (IEEE, New York, 2003), p. 3497.

[32] R. Bruce, R. W. Assmann, L. Lari, and S. Redaelli, in Proceedings of the Machine Protection Panel Workshop, Annecy, France, 2013, pp. 99-107.

[33] R. Bruce and S. Redaelli, in Proceedings of the 6th LHC Beam Workshop, Evian, France, 2015, pp. 203-210.

[34] J. Wozniak, V. Baggiolini, D. G. Quintas, and J. Wenninger, in Proceedings of ICALEPCS, Knoxville, Tennessee, USA (Jacow, Knoxville, 2007. 